\title{
Total Equitable Bondage Number of a Graph
}

\author{
S. K. Vaidya ${ }^{1 *}$, A. D. Parmar ${ }^{2}$ \\ ${ }^{1}$ Department of Mathematics, Saurashtra University, Rajkot - 360005, India \\ ${ }^{2}$ Atmiya Institute of Technology \& Science for Diploma Studies, Rajkot - 360005, India \\ Received 11 September 2017, accepted in final revised form 15 May 2018
}

\begin{abstract}
If for any total dominating set $D$ with $v \in V(G)-D$ there exists a vertex $u \in D$ such that $u v \in E(G)$ and $|d(v)-d(u)| \leq 1$ then $D$ is called the total equitable dominating set. The minimum cardinality of the total equitable dominating set is called the total equitable domination number denoted by $\gamma_{t}^{e}(G)$. The bondage number $b(G)$ of a nonempty graph $G$ is the minimum cardinality among all sets of edges $E_{0} \subseteq E(G)$ for which $\gamma\left(G-E_{0}\right)>\gamma(G)$. We introduced the concept of total equitable bondage number and proved several results.

Keywords: Dominating set; Equitable dominating set; Total dominating set; Bondage number.
\end{abstract}

AMS Subject Classification Number (2010): 05 C69

(C) 2018 JSR Publications. ISSN: 2070-0237 (Print); 2070-0245 (Online). All rights reserved. doi: http://dx.doi.org/10.3329/jsr.v10i3.33940

J. Sci. Res. 10 (3), 231-238 (2018)

\section{Introduction}

By a graph $G$ we mean a simple, finite and undirected graph $G$ with vertex set $V(G)$ and edge set $E(G)$. For any undefined term and notation in graph theory we rely upon West [13] while for standard terminology related to theory of domination we refer to Haynes et al. [4].

The set $D \subseteq V(G)$ of vertices in a graph $G$ is called dominating set if every vertex $v \in V(G)$ is either an element of $D$ or is adjacent to an element of $D$. The minimum cardinality of a dominating set of $G$ is called the domination number of $G$ which is denoted by $\gamma(G)$. There are various domination models like global domination [7], total domination [2], equitable domination [8], equi-independent equitable domination [9], total near equitable domination in graphs [6], etc.

The concept of total domination was introduced by Cockayne et al. [2] while the concept of equitable domination was introduced by Swaminathan and Dharmalingam [8]. Motivated through these concepts a new concept of total equitable domination was

* Corresponding author: samirkvaidya@yahoo.co.in 
conceived by Basavanagoud et al. [1] and further explored by Vaidya and Parmar [10-12]. The concepts of total dominating set and total equitable dominating set are useful for the formation of any committee. It is desirable that each committee member might feel comfortable knowing at least one member of the committee. In this situation total domination is useful while there is no difference of opinion between any two members or they differ on at most one issue. In this situation the concept of equitable domination is applicable. We have introduced the concept of total equitable bondage number.

Definition 1.1 A subset $D$ of $V(G)$ is called a total dominating set of $G$ if $N(D)=V(G)$ or equivalently if every vertex $v \in V(G)$ is adjacent to at least one element in $D$. The minimum cardinality of total dominating set is called total domination number which is denoted by $\gamma_{t}(G)$.

Definition 1.2 A subset $D$ of $V(G)$ is called an equitable dominating set if for every $v \in V(G)-D$ there exists a vertex $u \in D$ such that $u v \in E(G)$ and $|d(v)-d(u)| \leq 1$, where $d(u)$ denotes the degree of vertex $u$ and $d(v)$ denotes the degree of vertex $v$. The minimum cardinality of $D$ is called the equitable domination number of $G$ which is denoted by $\gamma^{e}(G)$.

Definition 1.3 A dominating set which is both total and equitable is called total equitable dominating set. The total equitable domination number $\gamma_{t}^{e}(G)$ of $G$ is the minimum cardinality of a total equitable dominating set of $G$.

We note that if $G$ is a disconnected graph with $n$-components $G_{l}, G_{2}, G_{3}, \ldots, G_{n}$ without isolated vertices and $G=\bigcup_{i=1}^{n} G_{i}$ then $\gamma_{t}^{e}(G)=\sum_{i=1}^{n} \gamma_{t}^{e}\left(G_{i}\right)$.

Definition 1.4 The bondage number $b(G)$ of a nonempty graph $G$ is the minimum cardinality among all sets of edges $E_{0} \subseteq E(G)$ for which $\gamma\left(G-E_{0}\right)>\gamma(G)$.

The concept of bondage number was introduced by Fink et al. [3] which is useful for measuring the vulnerability of the network under link failure. Many types of bondage numbers are introduced depending upon the characteristics of dominating sets. Some of them are total bondage number, roman bondage number, restrained bondage number are really noteworthy.

The concept of total bondage number was introduced by Kulli and Patwari [5]. The total equitable bondage number is a bondage number with the additional property that removal of an edge subset from the given graph results in a graph with larger total equitable domination number. This concept is also useful for measuring the link failure of network.

Definition 1.5 If $\gamma_{t}(G) \neq|V(G)|$ and $\left\langle G-E_{0}\right\rangle$ contains no isolated vertices then the total bondage number $b_{t}(G)$ of a graph $G$ is the minimum cardinality among all sets of edges $E_{0} \subseteq E(G)$ for which $\gamma_{t}\left(G-E_{0}\right)>\gamma_{t}(G)$.

Definition 1.6 If $\gamma_{t}^{e}(G) \neq|V(G)|$ and $\left\langle G-E_{0}\right\rangle$ contains no isolated vertices then the total equitable bondage number $b_{t}^{e}(G)$ of a graph $G$ is the minimum cardinality among all sets of edges $E_{0} \subseteq E(G)$ for which $\gamma_{t}^{e}\left(G-E_{0}\right)>\gamma_{t}^{e}(G)$.

We avoid the removal of two consecutive edges in $P_{n}$ and $C_{n}$ as it give rise to isolated vertices in the resultant graphs which is not an ideal situation for the discussion of total equitable bondage number. 
Definition 1.7 The wheel $W_{n}$ with $n$ vertices is defined to be the join of $K_{1}$ and $C_{n-1}$. The vertex corresponding to $K_{1}$ is known as apex while the vertices corresponding to $C_{n}$ are known as rim vertices.

We will state some of the existing results for our ready reference.

Proposition 1.8 [1] For any path $P_{n}$ with $n \geq 2$ vertices,

$\gamma_{t}^{e}\left(P_{n}\right)= \begin{cases}\frac{n}{2} & ; \text { if } n \equiv 2(\bmod 4) \\ \left\lfloor\frac{n}{1}\right\rfloor+1 & ; \text { otherwise }\end{cases}$

Proposition 1.9 [1] For any cycle $C_{n}$ with $n \geq 3$ vertices,

$\gamma_{t}^{e}\left(C_{n}\right)= \begin{cases}\frac{n}{2}+1 & ; \text { if } n \equiv 2(\bmod 4) \\ \left\lceil\frac{n}{2}\right\rceil & ; \text { otherwise }\end{cases}$

Proposition 1.10 [1] For any complete graph $K_{n}$ with $n \geq 2$ vertices, $\gamma_{t}^{e}\left(K_{n}\right)=2$.

Proposition 1.11 [1] For any complete bipartite graph $K_{m, n}$,

$\gamma_{t}^{e}\left(K_{m, n}\right)= \begin{cases}2 & ; \text { if }|m-n| \leq 1,1 \leq m \leq n \\ m+n & ; \text { if }|m-n| \leq 2, m, n \geq 2\end{cases}$

Proposition 1.12 [1] For any wheel $W_{n}$ with $n \geq 4$ vertices,

$\gamma_{t}^{e}\left(W_{n}\right)= \begin{cases}2 & ; \text { if } n=4,5 \\ \left\lceil\frac{n-1}{2}\right\rceil+1 & ; \text { otherwise }\end{cases}$

\section{Main Results}

Theorem 2.1 The total equitable bondage number of path of order $n \geq 3$ is given by

$b_{t}^{e}\left(P_{n}\right)= \begin{cases}2 ; & \text { if } n \equiv 2(\bmod 4) \\ 1 ; & \text { otherwise }\end{cases}$

Proof: If $\left\{\mathrm{e}_{1}, \mathrm{e}_{2}, \mathrm{e}_{3}, \ldots, \mathrm{e}_{\mathrm{n}-1}\right\}$ is the edge set of path $P_{n}$ then $H=P_{n}-\bigcup_{i=2}^{n-2}\left\{e_{i}\right\}$ is a disconnected graph without isolated vertices.

Case 1: For $n \neq 2(\bmod 4)$

The graph $\mathrm{H}$ obtained by removal of an edge $e_{2}$ from path $P_{n}$ then $\mathrm{H}$ is a graph with two components $P_{2}$ and $P_{n-2}$.

Also

$$
\begin{aligned}
\gamma_{t}^{e}(H) & =\gamma_{t}^{e}\left(P_{2}\right)+\gamma_{t}^{e}\left(P_{n-2}\right) \\
& =2+\left\lfloor\frac{n-2}{2}\right\rfloor+1 \\
& =\left\lfloor\frac{n}{2}\right\rfloor+2>\left\lfloor\frac{n}{2}\right\rfloor+1 \\
& >\gamma_{t}^{e}\left(P_{n}\right)
\end{aligned}
$$




\section{Total Equitable Bondage Number}

That is, $\gamma_{t}^{e}(H)>\gamma_{t}^{e}\left(P_{n}\right)$.

Hence, $b_{t}^{e}\left(P_{n}\right)=1$, when $n \neq 2(\bmod 4)$.

Case 2: For $n \equiv 2(\bmod 4)$

In this case the graph $H$ obtained by removal of an edge $e_{i}$ from path $P_{n}$ with two components $P_{i}$ and $P_{i-1}$.

Then, $\gamma_{t}^{e}(G)=\gamma_{t}^{e}\left(P_{i}\right)+\gamma_{t}^{e}\left(P_{n-i}\right)=\gamma_{t}^{e}\left(P_{n}\right)$. Therefore, $b_{t}^{e}\left(P_{n}\right) \neq 1$.

Now let the graph $H$ be obtained by removal of two edges precisely $e_{2}$ and $e_{n-2}$ from path $P_{n}$ then $H$ is a graph with three components $P_{2}, P_{n-4}$ and $P_{2}$.

Then,

$$
\begin{aligned}
\gamma_{t}^{e}(H) & =2 \gamma_{t}^{e}\left(P_{2}\right)+\gamma_{t}^{e}\left(P_{n-4}\right) \\
& =4+\frac{n-4}{2} \\
& =\frac{n}{2}+2 \\
& >\gamma_{t}^{e}\left(P_{n}\right)
\end{aligned}
$$

Hence, $b_{t}^{e}\left(P_{n}\right)=2$, when $n \equiv 2(\bmod 4)$.

Theorem 2.2 The total equitable bondage number of cycle of order $n \geq 4$ is given by $b_{t}^{e}\left(C_{n}\right)= \begin{cases}3 ; & \text { if } n \equiv 2(\bmod 4) \\ 2 ; & \text { otherwise }\end{cases}$

Proof: If $\left\{\mathrm{e}_{1}, \mathrm{e}_{2}, \mathrm{e}_{3}, \ldots, \mathrm{e}_{\mathrm{n}}\right\}$ is the edge set of cycle $C_{n}$ then $H=C_{n}-\left\{e_{i}\right\}$ is a path and $H=C_{n}-\bigcup_{i=2}^{n-2}\left\{e_{i}\right\}$ is a disconnected graph without isolated vertices.

Thus, $\gamma_{t}^{e}(H)=\gamma_{t}^{e}\left(C_{n}-e_{i}\right)=\gamma_{t}^{e}\left(P_{n}\right)$. Therefore, $b_{t}^{e}\left(C_{n}\right) \neq 1$.

Case 1: For $n \neq 2(\bmod 4)$

The graph $\mathrm{H}$ obtained by removal of two edges namely $e_{i}$ and $e_{i+2}$ for $i \in\{1,2,3, \ldots, n\}$ from $C_{n}$ is a graph with two components $P_{2}$ and $P_{n-2}$.

Then,

$$
\begin{aligned}
\gamma_{t}^{e}(H) & =\gamma_{t}^{e}\left(P_{2}\right)+\gamma_{t}^{e}\left(P_{n-2}\right) \\
& =2+\left\lfloor\frac{n-2}{2}\right\rfloor+1 \\
& =\left\lfloor\frac{n-2}{2}\right\rfloor+2\left\lceil\frac{n}{2}\right\rfloor \\
& >\gamma_{t}^{e}\left(C_{n}\right)
\end{aligned}
$$

Hence, $b_{t}^{e}\left(C_{n}\right)=2$, when $n \neq 2(\bmod 4)$. 
Case 2: The graph $H$ obtained by removal of three edges precisely $e_{i}, e_{i+2}$ and $e_{i+4}$ from $C_{n}$ is a graph with three components $P_{2}, P_{2}$ and $P_{n-4}$.

Then,

$$
\begin{aligned}
\gamma_{t}^{e}(H) & =2 \gamma_{t}^{e}\left(P_{2}\right)+\gamma_{t}^{e}\left(P_{n-4}\right) \\
& =4+\frac{n-4}{2} \\
& =\frac{n}{2}+2 \\
& >\gamma_{t}^{e}\left(C_{n}\right)
\end{aligned}
$$

Hence, $b_{t}^{e}\left(C_{n}\right)=3$.

Theorem 2.3 The total equitable bondage number of complete graph of order $n \geq 4$ is given by

$b_{t}^{e}\left(K_{n}\right)=\left\{\begin{array}{l}3 ; \text { if } n=4 \\ 2 ; \text { otherwise }\end{array}\right.$

\section{Proof:}

Case 1: For $n=4$

The graph $H$ obtained by removal of two edges then $\gamma_{t}^{e}(H)=2$. Therefore, $b_{t}^{e}\left(K_{4}\right) \neq 2$.

If there exists the graph $H$ obtained by removal of precisely three edges such that $\mathrm{H}$ is isomorphic to $K_{1,3}$. Thus $\gamma_{t}^{e}(H)=4$. Therefore $b_{t}^{e}\left(K_{4}\right)=3$.

\section{Case 2: For $n>4$}

In this case the graph $H$ obtained by removal of one edge then the graph it contains two vertices of degree $n-2$. Thus $\gamma_{t}^{e}(H)=2$. Consequently $b_{t}^{e}\left(K_{n}\right) \neq 2$.

If the graph $H$ obtained by removal of two adjacent edges, then it contains a vertex of degree $n-3$ which is not equitably adjacent to remaining vertices. Thus, $\gamma_{t}^{e}(H)=3$. Consequently, $b_{t}^{e}\left(K_{n}\right)=2$.

Theorem 2.4: The total equitable bondage number of wheel $W_{n}$ is given by

$$
b_{t}^{e}\left(W_{n}\right)= \begin{cases}1 ; & \text { if } n=5 \\ 3 ; & \text { if } n=4 \text { or } n \equiv 2(\bmod 3) \\ 2 ; & \text { otherwise }\end{cases}
$$

Proof: For the wheel $W_{n}$, let $\left\{u, v_{1}, v_{2}, \ldots, v_{n-1}\right\}$ be the set of vertices where $u$ is the apex and $e_{1}, e_{2}, \ldots, e_{n-1}$ are rim edges.

We consider the following cases:

\section{Case 1: For $n=5$}

The graph $H$ obtained by removal of an edge $e_{i}$ from $W_{5}$ then $\gamma_{t}^{e}(H)=\gamma_{t}^{e}\left(W_{5}\right)+1$. Hence $b_{t}^{e}\left(W_{5}\right)=1$.

Case 2: For $n=4$ and $n \equiv 2(\bmod 4)$. 
If the graph $H$ obtained by removal of two non-consecutive edges of $W_{n}$ then there exists a minimal total equitable dominating set of $H$ which is also a minimal total equitable dominating set of $W_{n}$. Therefore, $\gamma_{t}^{e}(H)=\gamma_{t}^{e}\left(W_{n}\right)$.

If the graph $H$ obtained by removal of two consecutive rim edges, precisely $e_{1}$ and $e_{2}$ from $W_{n}$ then minimal total equitable dominating set of $H$ is $\left\{u, v_{2}\right\} \bigcup\left\{v_{4}, v_{7}, \ldots, v_{n-1}\right\}$ with cardinality $\left\lceil\frac{n-1}{3}\right\rceil+1$. Therefore $\gamma_{t}^{e}(H)=\gamma_{t}^{e}\left(W_{n}\right)$. Consequently, $b_{t}^{e}\left(W_{n}\right)>2$.

The graph $H$ obtained by removal of three consecutive rim edges, precisely $e_{1}, e_{2}$ and $e_{3}$ from $W_{n}$ then minimal total equitable dominating set of $H$ is $\left\{u, v_{1}, v_{2}, v_{3}\right\} \bigcup\left\{v_{5}, v_{8}, \ldots, v_{n-3}\right\}$ with cardinality $\left\lceil\frac{n-1}{3}\right\rceil+2$. Therefore $\gamma_{t}^{e}(H)=\gamma_{t}^{e}\left(W_{n}\right)+1$.

Hence $b_{t}^{e}\left(W_{n}\right)=3$.

Case 3: For $n \equiv 0$ or $1(\bmod 3)$

If the graph $H$ obtained by removal of any edges $e$ of $W_{n}$ then there exists a minimal total equitable dominating set of $H$ which is also a minimal total equitable dominating set of $W_{n}$. Therefore, $\gamma_{t}^{e}(H)=\gamma_{t}^{e}\left(W_{n}\right)$.

If the graph $H$ obtained by removal of two consecutive rim edges, precisely $e_{1}$ and $e_{2}$ from $W_{n}$ then minimal total equitable dominating set of $H$ is $\left\{u, v_{1}, v_{2}\right\} \bigcup\left\{v_{3}, v_{7}, \ldots, v_{n-1}\right\}$ with cardinality $\left[\frac{n-1}{3}\right]+2$. Therefore $\gamma_{t}^{e}(H)=\gamma_{t}^{e}\left(W_{n}\right)+1$. Hence $b_{t}^{e}\left(W_{n}\right)=2$.

Theorem 2.5 The total equitable bondage number of complete bipartite graph $K_{m, n}$ with $|m-n| \leq 1$ is given by

$b_{t}^{e}\left(K_{m, n}\right)= \begin{cases}1 ; & \text { if }|m-n|=1 \\ 2 ; & \text { if }|m-n|=0\end{cases}$

\section{Proof:}

Case 1: For $|m-n|=1$

If the graph $H$ obtained by removal of an edge $e=u v$ from $K_{m, n}$, then $|d(u)-d(v)|=2$. Thus $\gamma_{t}^{e}(H)=\gamma_{t}^{e}\left(K_{m, n}\right)+1$. Hence $b_{t}^{e}\left(K_{m, n}\right)=1$.

\section{Case 2: For $|m-n|=0$}

If the graph $H$ obtained by removal of an edge $e=u v$ from $K_{m, n}$, then $|d(u)-d(v)|=2$. Thus $\gamma_{t}^{e}(H)=\gamma_{t}^{e}\left(K_{m, n}\right)$. Therefore, $b_{t}^{e}\left(K_{m, n}\right)>1$.

If the graph $H$ obtained by removal of two adjacent edges precisely $e_{1}=u v$ and $e_{2}=v w$ from $K_{m, n}$ then $|d(u)-d(v)|=2=|d(v)-d(w)|$. Thus $\gamma_{t}^{e}(H)=\gamma_{t}^{e}\left(K_{m, n}\right)+1$. Hence $b_{t}^{e}\left(K_{m, n}\right)=2$. 
Remark 2.6 For complete bipartite graph $K_{m, n}$ with $|m-n| \geq 2, \gamma_{t}^{e}\left(K_{m, n}\right)=m+n$ which is not favorable situation for the discussion of $b_{t}{ }^{e}\left(K_{m, n}\right)$

Theorem 2.7 If $\mathrm{T}$ is a tree except $K_{1, n-1}$ and $P_{n}$ with order $n>3$, where $n \equiv 2(\bmod 4)$. Then $b_{t}^{e}(T)=1$.

Proof: Let $T \neq K_{1, n-1}$ be any nontrivial tree of order $n>3$. To prove the result we consider following cases:

Case 1: If $u$ and $v$ are not equitable vertices in $V(T)$ then $\gamma_{t}^{e}(T)=n$ which is not ideal situation for the discussion of $b_{t}^{e}(T)$.

Case 2: If there exists equitable vertices $u$ and $v$ in $V(T)$ such that $u v=e \in E(T)$ then the graph $H$ is obtained by removal of an edge $e$ from $T$ then it leaves a forest with two components and each component is tree namely, $T_{1}$ and $T_{2}$.

Thus, $\gamma_{t}^{e}(H)=\gamma_{t}^{e}\left(T_{1}\right)+\gamma_{t}^{e}\left(T_{2}\right)>\gamma_{t}^{e}(T)$ one of $u$ and $v$ is not dominated by any vertices from $V(T)$. Hence $b_{t}^{e}(T)=1$.

Theorem 2.8 For any tree $T, b_{t}^{e}(T)=2$ if and only if $T=P_{n}$ with $n \equiv 2(\bmod 4)$.

Proof: Let $T$ be a path $P_{n}$ with $n \equiv 2(\bmod 4)$. By Theorem $2.1 b_{t}^{e}(T)=2$.

Conversely, let $b_{t}^{e}(T)=2$ and let $e_{1}=u v$ be an edge with $|d(u)-d(v)|>1$ and $d(u)>d(v)$. Then there exists an edge $e_{2}=u v$ such that $|d(u)-d(v)| \leq 1$. If the graph $H$ is obtained by removal of an edge $e_{2}=u v$ from $T$ the $H$ is a disconnected graph with two components $P_{i}$ and $T_{1}$ (where $i<n-3$ ).

Also $\gamma_{t}^{e}(H)=\gamma_{t}^{e}\left(P_{i}\right)+\gamma_{t}^{e}\left(T_{1}\right)=\gamma_{t}^{e}(T)+1$.

Consequently, $b_{t}^{e}(T)=1$. This contradicts our assumption. Therefore all the edges of $T$ are equitable edges. That is, $T=P_{n}$. Then by Theorem $2.1, b_{t}^{e}(T)=2$ for $n \equiv 2(\bmod 4)$.

\section{Conclusion}

The concepts of bondage number and total bondage number were introduced by Fink et al. [3] and Kulli and Patwari [6] respectively, while we have introduced the concept of total equitable bondage number. Total equitable bondage numbers of some standard graph families have been investigated and determined the bounds for the total equitable bondage number of trees.

\section{References}

1. B. Basavanagoud, V. R. Kulli, and V. V. Teli, J. Comput. Mathemat. Sci. 5, 235 (2014).

2. E. J. Cockayne, R. M. Dawes, and S. T. Hedetniemi, Networks 10, 211 (1980). https://doi.org/10.1002/net.3230100304 
3. J. F. Fink, M. S. Jacobson, L. F. Kinch, and J. Roberts, Discrete Math. 86, 47 (1990). https://doi.org/10.1016/0012-365X(90)90348-L

4. T. W. Haynes, S. T. Hedetniemi, and P. J. Slater, Fundamentals of Domination in Graphs (Marcel Dekker, New York, 1998).

5. V. R. Kulli and D. K. Patwari, The Total Bondage Number of A Graph, In: Adv. Graph Theory Ed. V. R. Kulli (Vishwa Inter. Publ., 1991) pp. 227-235.

6. A. M. Sahal and V. Mathad, Int. J. Mathemat. Combinatorics 3, 104 (2014).

7. E. Sampathkumar, J. Mathemat. Physical Sci. 23(5), 377 (1989).

8. V. Swaminathan and K. M. Dharmalingam, Kragujevac J. Math. 35(1), 191 (2011).

9. S. K. Vaidya and N. J. Kothari, J. Sci. Res. 7(3), 77 (2015). https://doi.org/10.3329/jsr.v7i3.23412

10. S. K. Vaidya and A. D. Parmar, J. Comput. Mathematica 1(1), 98 (2017).

11. S. K. Vaidya and A. D. Parmar, Int. J. Soft Computing 7(2), 103 (2017). https://doi.org/10.26708/IJMSC.2017.2.7.09

12. S. K. Vaidya and A. D. Parmar, Malaya J. Matematik 6(2), 375 (2018).

13. D. B. West, Introduction to Graph Theory (Prentice Hall, New Delhi, India, 2003). 